\title{
Approximations for End-to-End Delay Analysis in OBS Networks with Light Load
}

\author{
D. Morató, E. Magana, M. Izal and J. Aracil \\ Dept. de Automática y Computación, Universidad Pública de Navarra \\ Campus Arrosadia s/n, 31006 Pamplona, SPAIN \\ email:daniel.morato@unavarra.es
}

\begin{abstract}
In this paper we provide an analysis of end-to-end delay in OBS networks and a large deviations approximation. The analysis is based on an exponential approximation of the OBS router blocking time and on the assumption of Poisson arrivals in routers along the path from source to destination. On the other hand, a light load assumption is performed, namely, waiting time is mainly due to residual life of the output wavelengths and not to buffering.
\end{abstract}

Keywords: Optical Burst Switching, end-to-end delay.

\section{INTRODUCTION AND PROBLEM STATEMENT}

Optical Burst Switching (OBS) is a transfer mode that allows the transfer of several IP packets in a single burst. By doing so, the transmission constraints associatted to small packets in the optical domain are circumvented. On the other hand, a Burst Control Packet (BCP) is sent along the route between the source and destination and before the burst itself is released. This allows for unconfirmed "on-the-fly" resource reservation for the optical bursts. Thus, OBS is a transfer mode that is halfway between circuit switching and pure packet switching. In this paper, we will assumme that wavelenghts are reserved for exactly the burst transmission time (Just Enough Time - JET)[1].

If no wavelength is available, the incoming bursts will be blocked and a Fiber Delay Line (FDL) will be reserved using DR. If either no FDLs are available or the blocking time of the output port is larger than the delay time of the available FDLs then the burst will be dropped. Therefore, not only the burst dropping rate is influenced by the number of wavelengths, but also by their blocking time. In a previous paper [2] we show that the blocking time of an optical burst is exponentially distributed, regardless of the burst size distribution, and as long as the first moment is finite. In fact, a variety of burst size distributions may arise from the different burst assembly algorithms used at the (burstifier). Such burstifiers are functional units located at the edges of the optical network, that actually perform the grouping of several IP packets into a single burst. For example, the burst size turns out to be Gaussian for timer-based schemes [3]. Other non-Gaussian burst size distributions that have also been considered in the literature are the exponential distribution [4], [5], [6], the hyperexponential distribution [7], and the Pareto distribution [7]

Precisely, the above studies deal with the analysis of a single OBS router [4], [5], [6], [7], specially concerning blocking probability. In this paper, we will focus on the end-to-end performance of an OBS network comprising several routers in the route from source and destination. It is expected that time-constrained traffic, such as interactive video traffic, will be carried by the optical network. Furthermore, non-interactive services such as TCP services are also influenced by end-to-end delay, since loss detection is timer-based. Eventually, expiration of ACK timers can make the TCP connection enter congestion avoidance, implying severe throughput degradation. In conclusion, knowledge of the end-to-end delay performance of an OBS network is of fundamental importance for the deployment of time-constrained services over all-optical WDM networks.

\section{A. Network scenario}

Figure 1 shows the scenario under analysis. A number of $N$ OBS routers are traversed by the optical burst from the source burstifier (aggregation) to the destination burstifier (deaggregation). The delay incurred is the sum of the delay contributions at each OBS router.

The following assumptions will be made: 


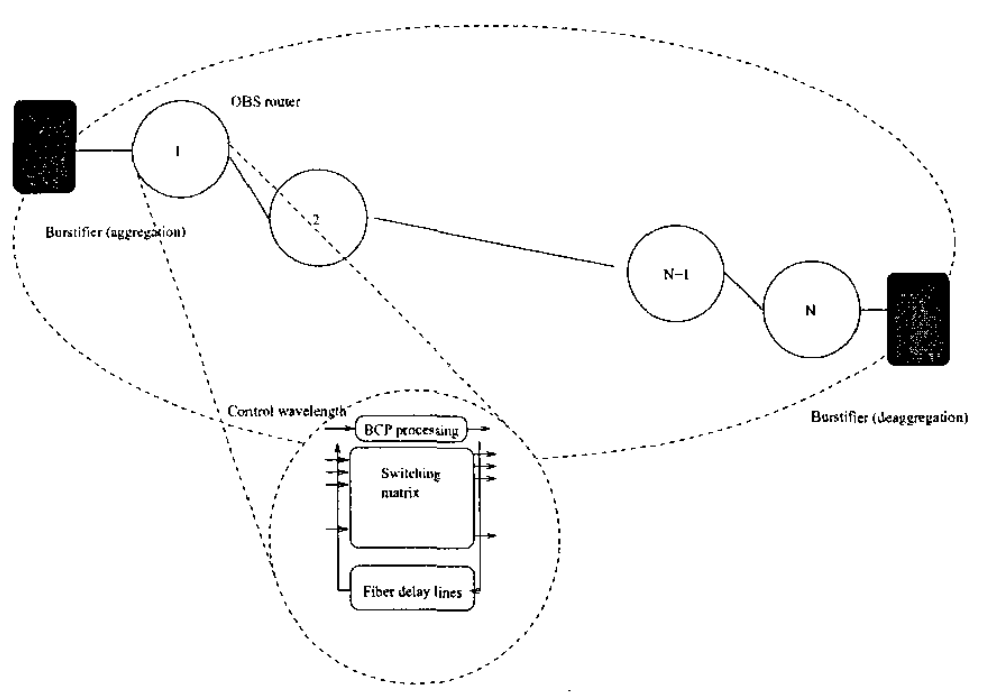

Fig. 1. Network scenario.

- The OBS routers have full wavelength conversion capability. Thus, an input burst will be blocked if and only if all wavelengths at the desired output port are occupied. Such blocking probability, regardless of the burst length distribution, is given by the Erlang-B formula [7].

- The routers along the path between source and destination remain stationary as far as the bloccking probability is concerned. As a result, the blocking probability along the path can be characterized by a vector $\left(p_{1}, \ldots, p_{N}\right)$. The blocking probability $p_{i}, i=1, \ldots, N$ is determined by the number of wavelengths at the output port and the traffic load.

- The time offset between BCP and burst remain constant along the path, i. e., the BCP is buffered to compensate for waiting times of blocked bursts.

- The number of fiber delay lines is large enough to accomodate any blocked burst and there is no recirculation. The FDL is also capable of providing any blocking time delay and it is variable length. Thus, as soon as any wavelength in the output port becomes available, the burst is immediately relayed to the free wavelength.

- The effect of waiting time in queue is limited to the residual life of the bursts in service. This a light-load approximation that simplifies the analysis.

With this assumptions in mind, the end-to-end delay is given by the sum of blocking times along the path form source to destination. In the following section, an analysis of the blocking time distribution is provided.

\section{BLOCKING TIME DISTRIBUTION}

Let us consider that the burst transmission time distribution has a finite first moment equal to $E X$. Let $R_{j}$ be the residual time in service (residual life) of the burst being transmitted in wavelength $j$, where $j=1, \ldots, M$, being $M$ the number of wavelengths per port (servers). The output port will be blocked if and only if $j=M$. Let $Y$ be the random variable that represents the blocking time for an incoming burst. We wish to derive $P(Y>y), y>0$, i.e. the survival function of the blocking time. Since burst arrivals are Poisson, and due to the PASTA property, the blocking time is given by the minimum of the residual lives of the $M$ bursts in service, namely $\{Y>y\}=\min _{j=1, \ldots, M}\left\{R_{j}>y\right\}$. Since bursts sizes are independent and identically distributed

$$
P(Y>y)=\prod_{j=1}^{M} P\left(R_{j}>y\right)=P\left(R_{1}>y\right)^{M}
$$

Equation (1) provides an expression for the blocking time distribution (survival function). Note that the blocking time distribution depends on the burst size distribution. Under the weak assumption of finiteness in the burst size first moment, we will show that the blocking time survival function becomes exponential with the number of wavelengths $(M)$.

\section{A. Approximations for the blocking time distribution (residual life)}

The density of the residual life of a burst in service $\left(P\left(R_{1}>y\right)\right)$, as seen by Poisson arrivals, can be obtained from the survival function of the service time as follows $[8, \mathrm{pp} .172$, vol. I] 


$$
\hat{f}_{R_{1}}(z)=\frac{P\left(X_{1}>z\right)}{E X_{1}}
$$

Thus,

$$
\begin{aligned}
& P\left(R_{1}>y\right)=\int_{y}^{\infty} \hat{f}_{R_{1}}(z) d z=\int_{0}^{\infty} \hat{f}_{R_{1}}(z) d z- \\
& -\int_{0}^{y} \hat{f}_{R_{1}}(z) d z=1-\frac{1}{E X_{1}} \int_{0}^{y} P\left(X_{1}>z\right) d z
\end{aligned}
$$

since $R_{1} \geq 0$ a. s. From the previous equation and (1)

$$
P(Y>y)=\left(1-\frac{1}{E X_{1}} \int_{0}^{y} P\left(X_{1}>z\right) d z\right)^{M}
$$

Since $P\left(X_{1}>z\right)$ is a monotonically decreasing function,

and

$$
\left(1-\frac{y}{E X_{1}}\right)^{M}<\left(1-\frac{1}{E X_{1}} \int_{0}^{y} P\left(X_{1}>z\right) d z\right)^{M}
$$

$$
\left(1-\frac{1}{E X_{1}} \int_{0}^{y} P\left(X_{1}>z\right) d z\right)^{M}<\left(1-\frac{y P\left(X_{1}>y\right)}{E X_{1}}\right)^{M}
$$

For values of $M$ and $y$ such that $M * P\left(X_{1}>y\right) \sim M$ (note that this happens for values of $y$ not in the tail of the distribution and for moderate to large number of wavelengths per port), (5) and (6) yield

$$
P(Y>y)=\left(1-\frac{1}{E X_{1}} \int_{0}^{y} P\left(X_{1}>z\right)\right)^{M} \approx e^{-\frac{M y}{E X_{1}}}
$$

In order to derive the last equation, it has been taken into account that $e^{-x}=1-x+o(x)$. This approximation is more accurate the smaller the value of $y$ and the larger the value of $N$. Further results are provided in [2], including exact expressions (eq. 1) for Pareto-distributed and Gaussian-distributed burst sizes.

\section{END-TO-END ANALYSIS}

In what follows, it will be assummed that all OBS routers will be assummed to have the same input traffic load, same number of ports and same number of wavelengths per port.

Let us denote by $X_{i}$ the random variable that provide the blocking time at OBS router $i, i=1, \ldots, N$. Let $Y=X_{1}+\ldots+X_{N}$ denote the end-to-end delay. Sincec the network is homogeneous the $X_{i} \mathrm{~s}$ are i.i.d and, following (7), the blocking time is exponential with rate $\lambda=M / E X$. Since the blocking time is null if the burst is not blocked, the density of $X_{1}$ is given by

$$
f_{X_{i}}(x)=p \lambda e^{-\lambda x} .
$$

where $p=p_{1}=\ldots=p_{N}$ is the blocking probability of any of the OBS routers along the path, due to the homogeneity assumption. In order to obtain the distribution of $Y$ let us condition to the event $A_{i}=$ \{exactly $i$ out of $N$ OBS routers are blocked $\}, i=1, \ldots, N$. Then, for any $a>0$

$$
P\left(X_{1}+\ldots+X_{N} \geq N a\right)=\sum_{i=0}^{N} P\left(X_{1}+\ldots+X_{N} \geq N a \mid A_{i}\right) P\left(A_{i}\right)
$$

and, since $P\left(A_{i}\right)$ is a binomial measure,

$$
P\left(X_{1}+\ldots+X_{N} \geq N a\right)=\sum_{i=0}^{N} P\left(X_{1}+\ldots+X_{N} \geq N a \mid A_{i}\right)\left(\begin{array}{c}
N \\
i
\end{array}\right) p^{i}(1-p)^{N-i} .
$$

In order to obtain an expression for the conditional probability, note that, since the $X_{i}$ s are i.i.d exponential random variables, then the partial sums $X_{1}+\ldots+X_{i}$ are Erlang random variables $E(\lambda, i)$. As a result,

$$
P\left(X_{1}+\ldots+X_{N} \geq N a\right)=\sum_{i=0}^{N} \int_{N a}^{\infty} \frac{\lambda^{i} e^{-\lambda x} x^{i-1}}{(i-1) !} d x\left(\begin{array}{c}
N \\
i
\end{array}\right) p^{i}(1-p)^{N-i}
$$

In the following section, we make use of large deviations techniques to provide a tail approximation. 


\section{LARGE DEVIATIONS APPROXIMATION}

By Chernoff's Theorem for i.i.d random variables

$$
P\left(X_{1}+\ldots+X_{N} \geq N a\right)=e^{-N l(a)+o(N)}
$$

being

$$
l(a)=\sup _{\theta}\left(\theta a-\log E e^{\theta X_{1}}\right) .
$$

Equation (12) can be used as an approximation for the tail of the end-to-end delay distribution, with large $N$. Let us first derive the moment generating function of $X_{1}$,

$$
E e^{\theta X_{1}}=(1-p) 0+p \int_{0}^{\infty} \lambda e^{(\theta-\lambda) x} d x=\frac{p \lambda}{\lambda-\theta}
$$

if $\theta<\lambda$. On the other hand, let us consider the map

$$
f(\theta)=\theta a-\log \left(\frac{p \lambda}{\lambda-\theta}\right)
$$

and let us obtain the maximum in the interval $\theta \in(0, \lambda)$. Such maximum is attained in $\theta^{*}=\lambda-1 / a$. Now, going back to (13) we obtain

$$
l(a)=f\left(\theta^{*}\right)=a \lambda-1-\log (a p \lambda)
$$

and, considering (12), it turns out that

$$
P\left(X_{1}+\ldots+X_{N} \geq N a\right) \approx \frac{1}{a p \lambda} e^{-N(a \lambda-1)}
$$

which approximates (11)

\section{CONCLUSIONS AND FUTURE}

In this paper we have provided an analysis and approximation of end-to-end delay in OBS networks, based on large deviations techniques and under a light-load assumption. The analysis is based on an exponential approximation of the blocking time distribution, which is insensitive to the burst distribution.

\section{REFERENCES}

[1] C. Qiao and M. Yoo. Optical burst switching (OBS) - A new paradigm for an optical Internet. Journal of High-Speed Networks, 8(1), 1999.

[2] D. Morato, M. Izal, J. Aracil, E. Maga na, and J. Miqueleiz. Blocking time analysis of OBS routers with arbitrary burst size distribution. In Proceedings of IEEE Globecom 2003, San Francisco, CA, December 2003.

[3] M. Izal and J. Aracil. On the influence of self-similarity in optical burst switching traffic. In Proceedings of IEEE Globecom 2002, Taipei, Taiwan, 2002.

[4] M. Yoo, C. Qiao, and S. Dixit, QoS performance of optical burst switching in IP over WDM networks. IEEE Journal of Selected Areas in Communications, 18(10):2062-2071, October 2000.

[5] S. Verma, H. Chaskar, and R. Ravikhant. Optical burst switching: A viable solution of terabit IP backbone. IEEE Network, November/December 2000.

[6] M. Yoo and C. Qiao. Supporting multiple classes of services in IP over WDM networks. In Proceedings of GLOBECOM 1999, Rio de Janeiro, Brazil, 1999.

[7] K. Dolzer, C. Gauger, J. Spath, and S. Bodamer. Evaluation of reservation mechanisms for optical burst switching. International Journal of Electronics and Communications (AE), 55(1), 2001.

[8] L. Kleinrock. Queueing Systems. John Wiley and Sons, 1975. 